\title{
From Next Generation Sequence to the Phenotype: Exploring the Bainbridge-Ropers Syndrome with Loss of Function Variants in ASXL3
}

\author{
Silvina Noemí Contreras-Capetillo'*, Melania Abreu-González ${ }^{2}$
}

'Laboratorio de Genética, Centro de Investigaciones Regionales Dr. Hideyo Noguchi, Mérida, Yucatán, México

${ }^{2}$ Laboratorio de Biología Molecular y Secuenciación Masiva. Genos Médica, Centro Especializado en Genética, Ciudad de México, México

Article Info

\section{Article Notes}

Received: July 16, 2018

Accepted: January 12, 2019

\section{*Correspondence:}

Dr. Silvina Noemi Contreras-Capetillo, Centro Regional de Investigaciones "Dr. Hideyo Noguchi", Universidad Autónoma de Yucatán. Calle $96 \mathrm{~s} / \mathrm{n} \times \mathrm{Av}$. Jacinto Canek y calle 47

Paseo de Las Fuentes. C.P. 97225 . Mérida, Yucatán, México; Telephone No: +52 (999) 9245809; Fax No: +52 (999) 923612; Email: silvina.contreras@correo.uady.mx

○ 2019 Contreras-Capetillo SN. This article is distributed under the terms of the Creative Commons Attribution 4.0 International License.

\section{Abstract}

In 2013, Bainbridge-Ropers syndrome (MIM \#615485) was described in patients with severe global developmental delay, postnatal microcephaly and feeding problems due to heterozygous loss of function variants in the ASXL3 gene. The ASXL3 is part of the ASXL gene family involved in gene expression during embryogenesis and they participate as epigenetic scaffolds capable of interacting with complex modifiers of chromatin and diverse transcription factors. Germline variants in ASXL1, ASXL2 and ASXL3 have been associated with neurodevelopmental disorders which clinical phenotypic presentation resembles to Bainbridge-Ropers syndrome thus elucidating these types of overlapping genetic disorders is challenging. Up to now, approximately forty patients have been confirmed with this syndrome by next generation sequencing. The implementation of whole exome sequencing allows early identification and definitive diagnosis of patients with clinically unestablished phenotypes, as seen in $A X L 3$ gene. This review discusses clinical and molecular features of variants in $A X L 3$ gene associated with Bainbridge-Ropers syndrome.

\section{Introduction}

Neurodevelopmental disorders without specific phenotype are highly undiagnosed, fortunately, next-generating sequencing (NGS) platforms are now able to confirm clinical phenotypes that in the past remained unknown ${ }^{1}$. These types of technologies have allowed for the discovery of new genes and new associations with diseases. In 2011, Mathew Bainbridge, a computational biologist, identified an abnormal copy of the ASXL3 gene in a patient who, after multiple genetic tests remained without diagnosis. After collaboration between Bainbridge and other experts in NGS, three additional individuals with variants in ASXL3 gene associated with neurodevelopment disease were found. Finally, in 2013 Bainbridge and colleagues reported $A S X L 3$ variants in four patients with similar clinical features to Bohring-Opitz syndrome (MIM *612990, BOS) without the classic posture. Global development delay, postnatal microcephaly and feeding difficulties with heterozygous loss of function mutations in ASXL3 gene (MIM *615115) were detected by whole-exome sequencing (WES) and whole genome sequencing (WGS) ${ }^{2}$. These findings have led to the identification of nearly forty patients in large scale NGS project (Deciphering Developmental Disorders Study, www.ddduk.org) ${ }^{3}$, cohorts of clinical exome (CES) ${ }^{4,5}$ or isolated cases of nonspecific developmental delay ${ }^{6,7}$ which required WES or CES as molecular approaches.

\section{Additional Sex Combs-Like Family (ASXL)}

The ASXL3 gene is part of the ASXL (Additional Sex Combs-Like) 
family in vertebrates (ASXL1, ASXL2 and ASXL3 genes) that encodes regulatory proteins of the trithorax and polycomb enhancers. The ASXL modulates the expression of homeotic genes during embryogenesis and functions as epigenetic scaffolds capable of interacting with complex modifiers of chromatin and diverse transcription factors ${ }^{8}$. ASXL1, ASXL2 and ASXL3 genes present common domains (ASXN, ASXH, ASXM1, ASXM2 and one PHD-finger) with thirteen exons and twelve introns ${ }^{9}$. The ASXL1, ASXL2 and $A S X L 3$ genes express ubiquitously but, $A S X L 3$ is predominantly expressed in the brain. These genes are involved in hereditary neurological disorders and the somatic presentation is associated with different types of cancers ${ }^{8}$. As more patients are described, it will be possible to determine if there is an increased risk of developing cancer in constitutional carriers.

Previously, studies have shown that ASXL1 gene is primarily involved in transcriptional activation and repression. In addition, somatic nonsense and frameshift variants have been frequently described in myelodysplastic syndrome and other hematological malignancies since, ASXL1 is essential for erythroid development and differentiation $^{10}$. Germline de novo ASXL1 variants in PHD and ASXM2 domains have shown to cause BOS ${ }^{9,11}$.

Dominant-negative effect variants in ASXL2 gene have recently been linked to neurodevelopmental disorder (Shashi-Pena syndrome; MIM \#617190, SHAPNS), as presented in six unrelated probands with de novo truncating variants. Reported patients demonstrated common clinical characteristics including delayed psychomotor development, variable intellectual disability, macrocephaly, prominent eyes, arched eyebrows, hypertelorism, glabellar nevus flammeus and hypotonia ${ }^{12}$.

Furthermore, pathogenic truncating de novo loss of- function ${ }^{4}$ variants in ASXL3 gene are associated with Bainbridge-Ropers syndrome (BRS). Frameshifts included $62 \%$ of the variants described, nonsense were reported in $34 \%$ and one report a splicing variant was identified $(0.03 \%)^{6}$ (Table 1$)$. These types of changes are consistent with the haploinsufficiency mechanism as proposed previously ${ }^{4}$. Missense variants were also reported in ASXL3 associated within the autistic spectrum ${ }^{13,14}$. Three variants presented recurrence in BRS. The c.3106C $>\mathrm{T}(\mathrm{p}$. Arg1036* $)^{4,14,15}$, present in both BRS and in an individual with autism spectrum disorder ${ }^{16}$. The c.4330C $>\mathrm{T}$ (p. Arg1444* $)^{3,5}$ and the $.3039+1 \mathrm{G}>\mathrm{A}^{6,15}$ which were described in unrelated patients (Table 1). In 2013, Bainbridge and colleagues generated the polarity hypothesis in relation to the location of the mutations associated with BRS. This suggested that truncated variants, which occur at end 5 'of the gene, are associated with a more severe phenotype. Nonetheless, this hypothesis was eliminated ${ }^{5,6,7}$ after accumulated evidence was examined. Despite the fact that the majority of the mutations are located on the largest exon, as in $A S X L 1^{17}$, to date, two clusters of variants have been proposed both equally affected $(\sim 43 \%)$ (Table 1$)$. One in the N-terminal region located between the codons 404 to 659 and another in the C-terminal (functional domain of chromatin-DNA recognition) between the codons 1045 to $1444^{6,17}$. Additionally, transcriptomes of fibroblasts from patients with BRS demonstrated $>500$ genes involved in transcriptional regulation, development and proliferation of the differentially expressed genes, establishing that BRS is associated with defects in transcriptional regulation ${ }^{3,5}$.

Of the 32 variants described in Table 1, 90\% were detected by WES. This was likely due to a higher diagnostic yield compared to CES or other NGS gene panels for neurodevelopmental disorders ${ }^{21}$. Based on these findings, it is necessary to include the ASXL gene family in NGS analysis in patients with subtle dysmorphism and psychomotor delay.

Recently, a compound heterozygous patient with BRS like features and primary IGF1 deficiency which presented the genotype c. $(2965 \mathrm{C}>\mathrm{G}) ;(3078 \mathrm{G}>\mathrm{C})$ was reported. It was proposed that the additively effect of both changes impacted in ASXL3 gene function. Although, in silico analysis suggests that the variant c. $2965 C>G$ was responsible for the loss of function. Functional studies will be necessary to confirm the real pathogenicity of the c. $2965 \mathrm{C}>\mathrm{G}$ rare variant ${ }^{22}$.

\section{Clinical features}

To date, fewer than 40 patients with variants in ASXL3 with the BRS phenotype have been reported $3,4,5,6,7,12,13,14,5$, 19. Usually, BRS presents as de novo truncating mutations in all patients with the exception of two siblings with the same mutation, which was not present in their parents. Germline mosaicism was not ruled out in this case. BRS patients are described from the prenatal stage ${ }^{18}$ to the age of $47^{20}$ with consistent phenotype features such as facial dysmorphisms with a broad and prominent forehead, arched eyebrows, downslanting palpebral fissures and anteverted or hypoplastic nares. These characteristics are shown to be present in less than $50 \%$ of the patients described. The principal manifestations are those related with neurological development. Global development delay, intellectual disability, from moderate to severe, late or absent language skills and hypotonia are present in more than $80 \%$ of the affected individuals. Feeding difficulties are also prevalent among these patients and more frequent during the neonatal period. ${ }^{2,3,5}$, (Table 2).

Both BOS and SHAPNS show intellectual disability and neurodevelopmental delay as a main characteristic. However, development delay is mild in SHAPNS compared to the others $^{2,4,13}$. BOS was first described in patients with feeding problems with primary or secondary microcephaly and severe neurodevelopmental delay. "BOS posture" consistent 
Table 1. Loss of function variants associated with BRS phenotype.

\begin{tabular}{|c|c|c|c|c|}
\hline NM_030632.2 & NP_085135.1 & Cluster & BRS Phenotype & Reference \\
\hline \multicolumn{5}{|c|}{ Nonsense Variants n=11/32 (34\%) } \\
\hline c. $1074 \mathrm{~T}>\mathrm{A}$ & p.Tyr358* & $\mathrm{N}$ & + & 3 \\
\hline c. $1210 \mathrm{C}>\mathrm{T}$ & p.Gln404* & $\mathrm{N}$ & + & 2 \\
\hline c.1369G $>\mathrm{T}$ & p.Glu457* & $\mathrm{N}$ & + & 4 \\
\hline c. $1396 C>T$ & p.Gln466* & $\mathrm{N}$ & + & 2 \\
\hline c. $1783 C>T$ & p.Gln595* & $\mathrm{N}$ & + & 3 \\
\hline c. $3106 C>T$ & p.Arg1036* & & + & $4,14,15$ \\
\hline c. $3364 C>T$ & p.Gln1122* & $\mathrm{C}$ & + & 5 \\
\hline c. $3613 G>T$ & p.Glu1205* & $\mathrm{C}$ & + & 4 \\
\hline c.3635T>G & p.Leu1212* & C & + & 3 \\
\hline c. $4144 C>T$ & p.Gln1382* & C & + & 3 \\
\hline c. $4330 \mathrm{C}>\mathrm{T}$ & p.Arg1444* & $\mathrm{C}$ & + & 3,5 \\
\hline \multicolumn{5}{|c|}{ Indels (Frameshift Variants) $n=20 / 32$ (62\%) } \\
\hline c.1082dup & p.Leu362Alafs*23 & & + & 3 \\
\hline c.1201del & p.Ala401Glnfs*8 & & + & 3 \\
\hline c.1219delA & p.Ser407Alafs*2 & $\mathrm{N}$ & + & 4 \\
\hline c.1314_1316delinsA & p. Ser439Argfs*7 & $\mathrm{N}$ & $\begin{array}{l}+ \text { and hyperventilation } \\
\text { induced athetosis }\end{array}$ & 8 \\
\hline c.1318dup & p.Glu440Glyfs*7 & $\mathrm{N}$ & $\begin{array}{c}+ \text { and pontocerebelar } \\
\text { hypoplasia }\end{array}$ & 18 \\
\hline c.1422dup & p.Pro $475^{*}$ & $\mathrm{~N}$ & + & 2 \\
\hline c.1448dupT & p.Thr484Asnfs*5 & $\mathrm{N}$ & + & 5 \\
\hline c.1484insTGAA & p.Asp $497^{*}$ & $\mathrm{~N}$ & + & 3 \\
\hline c.1491dup & p.Asn498* & $\mathrm{N}$ & + & 3 \\
\hline c.1897_1898delCA & p.Gln633Valfs*13 & $\mathrm{N}$ & + and trigonocephaly & 19 \\
\hline c.1978_1981deIGACA & p.Asp660Asnfs*1 & & + & 2 \\
\hline c.2992_2995del & p.Glu998Lysfs*26 & & + & 7 \\
\hline c.3028delC & p.Pro1010Leufs*14 & C & $\begin{array}{c}\text { + and prominence of the } \\
\text { Sylvian Fissure }\end{array}$ & 13 \\
\hline c.3127_3128dup & p.Gly1045Valfs*99 & C & + & 3 \\
\hline c.3178dup & p.Arg1060Profs*50 & C & + & 3 \\
\hline c.3313_3316delCAGA & p.Thr1106Argfs*36 & $\mathrm{C}$ & + & 15 \\
\hline c.3355dup & p.His1119Profs*7 & $\mathrm{C}$ & + & 3 \\
\hline c.3494_3495delGT & p.Cys $1165^{*}$ & $\mathrm{C}$ & + & 4 \\
\hline c.4072_4073delGT & p.Val1358Leufs*8 & C & + & 4 \\
\hline c.6697_6710dup & p.Ser2238Thrfs*3 & & + & 20 \\
\hline \multicolumn{5}{|c|}{ Splicing $1 / 32(0.03 \%)$} \\
\hline c. $3039+1 G>A$ & $?$ & C & + & 6,15 \\
\hline
\end{tabular}

to exrotation and/or adduction of the shoulders, flexion at the elbows, flexion at the wrists, and ulnar deviation of the wrists and/or fingers at the metacarpophalangeal joints, was described in those patients. This phenotype was associated to ASXL1 gene variants and was classified as a distinct condition from BRS, based on the absence of BOS posture in patients with ASXL3 gene variants ${ }^{23,24}$. SHAPNS is described as a neurological disease caused by ASXL2 gene variants. The overlapping features are identified by global developmental delay and feeding problems. But, macrocephaly instead of microcephaly seems to be SHAPNS most distinctive clinical sign ${ }^{12}$.

\section{Conclusion}

The relevance of the discovery of BRS and the diagnostic route lies in the reversibility of the daily routine of the patient with genetic conditions ${ }^{2}$, given that the majority of these patients have been diagnosed through molecular findings, which subsequently allowed them to be grouped in a similar phenotype. Clinical differences within the ASXL were identified between the ASXL1, ASXL2 and AXL3 genes phenotype. Differences in epigenetic regulating function factors that contribute to phenotype and temporality of the presentation of gene variants (germinal or somatic) could play an important role ${ }^{14}$. Primarily, described ASXL3 gene phenotypic variants have been classified as de novo. However, findings support a $<1 \%$ possibility of recurrency in germline mosaicism respectively.

Five years after the initial BRS description, it has 
Table 2. Clinical characteristic of 36 patients with variants in ASXL3 compared with phenotype reported in pathological variants in ASXL2 and ASXL1 in the literature.

\begin{tabular}{|c|c|c|c|}
\hline Phenotype & $\operatorname{ASXL3}(\mathrm{n}=36)$ & $\operatorname{ASXL2}(n=6)^{12}$ & $\operatorname{ASXL1}(\mathrm{n}=7)^{17}$ \\
\hline Age & Prenatal-47 years & 11 months -31 years & Neonatal-14 years \\
\hline \multicolumn{4}{|c|}{ Neurological } \\
\hline Global developmental delay/Intellectual disability & $100 \%$ & $100 \%$ & $100 \%$ \\
\hline Late/absent language skill & $92 \%$ & $33 \%$ & NR \\
\hline Hypotonia & $83 \%$ & $100 \% *$ & $21 \%$ \\
\hline Feeding difficulties & $75 \%$ & $100 \%$ & $100 \%$ \\
\hline Autism spectrum disorder/Autism & $47 \%$ & NR & NR \\
\hline Seizures & $39 \%$ & $83 \%$ & $50 \%$ \\
\hline Brain abnormalities & & $83 \%$ & $71 \%$ \\
\hline \multicolumn{4}{|c|}{ Craneofacial features. } \\
\hline Microcephaly & $36 \%$ & 0 & $100 \%$ \\
\hline Macrocephaly & 0 & $100 \%$ & 0 \\
\hline Downslanting palpebral fissures & $50 \%$ & 0 & 0 \\
\hline Broad/prominent forehead & $42 \%$ & $16 \%(1)$ & 0 \\
\hline Glabellar nevus flammeus & 0 & $100 \%$ & $86 \%$ \\
\hline Arched eyebrows & $31 \%$ & $100 \%$ & 0 \\
\hline Low-set/ posteriorly rotated ears & $22 \%$ & $100 \%$ & $43 \%$ \\
\hline Anteverted nares/hypoplastic nares & $22 \%$ & $16 \%(1)$ & $29 \%$ \\
\hline Ocular hypertelorism & $19 \%$ & $100 \%$ & $57 \%$ \\
\hline Upslanting palpebral fissures & $8 \%$ & 0 & $57 \%$ \\
\hline \multicolumn{4}{|c|}{ Systemic features } \\
\hline Ulnar deviation & $17 \%$ & 0 & 0 \\
\hline Hypertrichosis & $16 \%$ & $16 \%(1)$ & $86 \%$ \\
\hline BOS posture & 0 & 0 & $100 \%$ \\
\hline \multicolumn{4}{|c|}{ Others } \\
\hline & $\begin{array}{c}\text { Neonatal macro- } \\
\text { somy in one patient }\end{array}$ & $\begin{array}{l}\text { Structural cardiopathy, hypogli- } \\
\text { cemia }\end{array}$ & $\begin{array}{l}\text { Atresia choanal, atrial septal } \\
\text { defect, hepatomegaly, tho- } \\
\text { racolumbar scoliosis, ocular } \\
\text { abnormalities }\end{array}$ \\
\hline
\end{tabular}

been possible to provide answers to an ever-increasing number of families thanks to the significant achievement of obtaining a definite genetic diagnosis. The phenotypic definition associated with the ASXL gene family presents the challenge of the importance to use massive sequencing analysis in order to obtain more timely diagnosis associated with neurodevelopmental disorders.

\section{References}

1. Lee H, Deignan JL, Dorrani N, et al. Clinical exome sequencing for genetic identification of rare mendelian disorders. JAMA. 2014; 312: 1880-1887.

2. Bainbridge MN, Hu H, Muzny DM, et al. De novo truncating mutations in ASXL3 are associated with a novel clinical phenotype with similarities to Bohring-Opitz syndrome. Genome Med. 2013 Feb; 5: 1-9.

3. Balasubramaninan M, Willoughby J, Fry AE, et al. Delineating the phenotypic spectrum of Bainbridge-Ropers syndrome: 12 new patients with de novo, heterozygous, loss-of-function mutations in ASXL3 and review of published literature. J Med Genet. 2017; 8: 1-7.

4. Kuechler A, Czeschik JC, Graf E, et al. Bainbridge-Ropers syndrome caused by loos-of-function variants in ASXL3: a recognizable condition. Eur J Hum Genet. 2017 Feb; 25: 183-191.
5. Srivastava A, Ritesh KC, Tsan YC, et al. De novo dominant ASXL3 mutations alter H2A deubiquitination and transcription in BainbridgeRopers syndrome. Hum Mol Genet. 2016 Feb; 25(3): 597-608.

6. Hori I, Miya F, Ohashi K, et al. Novel splicing mutation in the ASXL3 gene causing Bainbridge-Ropers syndrome. Am J Med Genet Part A. 2016; 170: 1863-1867.

7. Contreras-Capetillo SN, Vilchis-Zapata ZH, Ribbon-Conde J, et al. Global developmental delay and postnatal microcephaly: BainbridgeRopers syndrome with a new mutation in ASXL3. Neurología. 2018 Sep; 33(7): 484-486.

8. Dad R, Walker S, Scherer SW, et al. Hyperventilation-athetosis in ASXL3 deficiency (Bainbridge-Ropers) syndrome. Neurol Genet. 2017 Oct; 3(5).

9. Katoh M. Functional and cancer genomics of ASXL family members. Br J Cancer. 2013; 109(2): 299-306.

10. Hilgendorf S, Folkerts H, Schuringa JJ, et al. Loss of ASXL1 triggers an apoptotic response in human hematopoietic stem and progenitor cells. Exp Hematol. 2016; 44: 1188-1196.

11. Hoischen A, M van Bon BW, Rodríguez-Santiago B, et al. De novo nonsense mutation in ASXL1 cause Bohring-Opitz syndrome. Nature Genetics 2011; 43(8): 729-731. 
12. Sashi V, Pena LMD, Kim K, et al. De novo truncating variants in ASXL2 are associated with a unique and recognizable clinical phenotype. Am J Hum Genet. 2016 Oct; 99(4): 991-999.

13. Chinen Y, Nakamura S, Ganaha Am, et al. Mild prominence of the Sylvian fissure in a Bainbridge-Ropers syndrome patient with a nove frameshift variant in ASXL3. Clin Case Rep. 2017 Dec; 6(2): 330-336.

14. Koboldt DC, Mihalic Mosher T, Kelly BE, et al. A de novo nonsense mutation in ASXL3 shared by siblings with Bainbridge-Ropers syndrome. Cold Spring Harb Mol Case Stud. 2018 Jun; 4(3).

15. Myers KA, White SM, Mohammed S, et al. Childhood-onset generalized epilepsy in Bainbridge-Ropers syndrome. Epilepsy Res. 2018 Feb; 140: 166-170.

16. De Rubeis S, He X, Goldberg AP, et al. 10. Synaptic, transcriptional, and chromatin genes disrupted in autism. Nature. 2014 Nov; 515(7526): 209-215.

17. Hoischen A, van Bon BW, Rodríguez-Santiago B, et al. De novo nonsense mutations in ASXL1 cause Bohring-Opitz syndrome. Nat Genet. 2011 Jun; 43(8): 729-31.

18. Bacrot $\mathrm{S}$, Mechler $\mathrm{C}$, Talhi N, et al. Whole exome seuencing diagnoses the first fetal case of Bainbridge-Ropers syndrome presenting as pontocerebellar hypoplasia type 1. Birth Defects Res. 2018 Apr; 110(6): 538-542.
19. Dinwiddie DL, Soden SE, Saunders CJ, et al. De novo frameshift mutation in ASXL3 in a patient with global developmental delay, microcephaly, and craniofacial anomalies. BMC Med Genomics. 2013 Sep; 6: 32.

20. Verhoeven W, Egger J, Rakers E, et al. Phenotypic characterization of an older adult male with late-onset epilepsy and a novel mutation in ASXL3 shows overlap with the associated Bainbridge-Ropers syndrome. Neuropsychiatr Dis Treat. 2018; 14: 867-870.

21. Srivastava $S$, Cohen SJ, Vernon $H$, et al. Clinical whole exome sequencing in child neurology practice. Ann Neurol. 2014 Oct; 76(4): 473-83.

22. Giri D, Rigden D, Didi M, et al. Novel compound heterozygous ASXL3 mutation causing Bainbridge-Ropers like syndrome and primary IGF1 deficiency. Int J Pediatr Endocrinol 2017; 8: 1-6.

23. Hastings R, Cobben JM, Gillessen-Kaesbach G, et al. Bohring-Opitz (Oberklanid-Danks) syndrome: clinical study, review of the literature, and discussion of possible pathogenesis. Eur J Hum Genet. 2011; 19: 513-519.

24. Magini P, Della Monica M, Giovannucci Uzielli ML, et al. Two novel patients with Bohring-Opitz syndrome caused by de novo ASXL1 mutations. Am J Med Genet A. 2012; 158A(4): 917-21. 